\title{
Proposal Of New Conjecture For Solution Of Goldback's Puzzle
}

\author{
Umasankar Dolai \\ Assistant teacher, Dwarigeria Pry. School, Garhbeta South C.L.R.C., Satbankura - 721253 \\ Dist. - Paschim Medinipur, West Bengal, India
}

\begin{abstract}
New conjecture about prime numbers is proposed for solving the criterion of Goldback's puzzle about natural numbers. It is found that Goldback's puzzle is a corollary of that new conjecture.

Keywords - Goldback's Puzzle, Prime Numbers Distribution, Remarks.

\section{Introduction}

In general mathematics, Goldback's puzzle about natural numbers is an unsolved problem till now. i.e. Every even number in natural numbers series is at least an addition of a pair of prime numbers except 2. e.g. $4=2+2,6=3+3,8=3+5,10=3+7,12=5+7$ etc. Here $2=1+1$; where 1 is not a prime number.It is seen that the truth of this puzzle can depend on a new conjecture drawn about the prime numbers. Moreover if this conjecture be proved, then Goldback's puzzle can serve as a corollary of that conjecture.
\end{abstract}

\section{New Conjecture}

Here a new conjecture about the prime numbers can be introduced to deduce the reality of Goldback's puzzle about natural numbers. The new conjecture is "there is at least a prime-prime distribution at a same distance in both sides from a number of natural numbers series except 1,2 and 3". The context of discussion will be clear if some examples are applied. e.g. The prime-prime distributions from the numbers 4,5 and 6 are given below.

Thus in this respect, it can be written as 3, 5 from 4; 3, 7 from 5 and 5, 7 from 6 . The differences 4 $3=1,5-4=1$ in case of $4 ; 5-3=2,7-5=2$ in case of 5 and $6-5=1,7-6=1$ in case of 6 . In both cases of 4,5 or 6 , the differences are same. i.e. There is a prime-prime distribution at a same distance in both sides from 4,5 or 6 . This criteria is also true for the other numbers of natural numbers series above those numbers. i.e. 3,11 for $7 ; 3,13$ or 5,11 for $8 ; 5,13$ or 7,11 for 9 etc.

\section{Discussion Of Natural Numbers Property}

It is a natural mathematical fact that every even number in natural number series is the additions of pairs of the numbers situated at a same distance in both sides from its half numbers. e.g. The addition-distributions of the even numbers 8,10 and 12 are obtained at a same distance in both sides from the numbers 4 (half of 8 i.e. $8=4+4$ ), 5 (half of 10 i.e. $10=5+5$ ) and 6 (half of 12 i.e. $12=6+6$ ) as the pairs of natural numbers (odd-odd and even-even).

\section{Number Figures And Number Tables}

The above topics will be more clear by the following number figures and number tables. i.e.
(i) $8=1+7$
$=2+6$
(ii) $10=1+9$
$=2+8$
$=3+7$
(iii) $12=1+11$
$=2+10$
$=3+9$
$=4+6 \quad=4+8$
$=3+5$$$
=5+7
$$

\section{Conclusion}

Again all even numbers are twice of the numbers of natural number series. i.e. The even numbers $=2 \mathrm{x}$ natural numbers $=2 \mathrm{x}\{1,2,3,4, \ldots \ldots \ldots \ldots \ldots \ldots . . .$. Thus all even numbers have the above types of distributions. Now if the new discussed conjecture about prime numbers be prove, then there must be the addition distributions of the pairs of prime numbers for all even numbers in natural numbers series. Because according to the new conjecture, there must be at least one prime-prime distribution at a same distance in both sides from a number of natural numbers series. Here $8=3+5,10=3+7$ and $12=5+7$. This is nothing but the same opinion stated in Goldback's puzzle.

However the even numbers 4 and 6 are not covered under this discussed conjecture because of the exceptional behaviour of the numbers 2 and 3 in this respect. Here
(a) $4=1+3$
(b) $6=1+5$ 


$$
=2+4
$$

Where there are no prime-prime distributions in the above cases with respect to the new proposed conjecture. But the other cases of Goldback's puzzle can be successfully explained by this new conjecture.

Note added in proof. It is proved that "there is exist at least a prime number in between a number and its twice in natural numbers series". e.g. 3 in between 2 and 4. Again it is known that "a number is situated at half distance from (with respect to) its twice at opposite side in natural number series". e.g. 4 is situated at half distance from its twice 8 at opposite side (i.e. $4=1 / 2 \times 8$ ). Thus the conclusion can be assumed from above opinions: "a number is situated at half distance from at least the prime number exist in between the number and its twice to another prime number at opposite side in natural numbers series". e.g. 5 is at least the prime number in between 4 and its twice 8 as well as 4 is exist in half distance from 8 at opposite side. Now 4 is situated at half distance from 5 to another prime number 3. i.e. 5-3=2; again 4-3=1 and 5-4=1. Here 1 is half of 2 .

The first prime number in between a number and its twice is 3. i.e. 3 is in between 2 and its twice 4 . There is no prime number in between 1 and its twice 2 . So the above assumed conclusion is valid only from number 4 . The numbers 1,2 and 3are exceptional in this case.

\section{Acknowledgements}

I wish to thank Mr. Pathik Guha for his essay about prime numbers in Bengali magazine "Anandamela". I indebt to thank Shree Gourisankar Dolai, student of higher secondary school for his help to type this paper. I also like to thank Mr. Sandip Maity, Assistant Engineer, WBSETCL for his inspiration to write this article by me.

\section{References}

[1] Paolo Giordano, The Solitude of Prime Numbers, Pamela Dorman Books (2010).

[2] Richard E. Crandall, Prime Numbers : A Computational Perspective, Springer (2005).

[3] Ribenboim, Paulo, The book of Prime Number Records, Sringer (1996).

[4] Matthew Watkins, Matt Tweea, Math Book: The Mystery of the Prime Numbers, Murray (2011). 